\title{
Bias-Corrected Maximum Likelihood Estimation of the Parameters of the Modified Power Function Distribution
}

\author{
Suttida Sangpoom $^{1}$ and Yuwadee Klomwises ${ }^{2, *}$ \\ ${ }^{I}$ Center of Excellence in Data Science for Health Study, Division of Mathematics and Statistics, \\ School of Science, Walailak University, Nakhon Si Thammarat 80160, Thailand \\ ${ }^{2}$ Department of Statistics, School of Science, King Mongkut's Institute of Technology Ladkrabang, \\ Bangkok 10520, Thailand
}

('Corresponding author's e-mail: yuwadee.kl@kmitl.ac.th)

Received: 26 February 2021, Revised: 20 May 2021, Accepted: 26 May 2021

\begin{abstract}
One of the extended power function distributions is the modified power function distribution. It has a malleable probability distribution and may be used to represent bounded data on an interval $(0,1)$. The maximum likelihood estimation (MLE) approach was used in the literature to estimate the distribution's parameters. However, because of the current prevalence of bias for a small sample size, this type of estimator has been widely warned. Consequently, we emphasize the method for reducing biased of the maximum likelihood estimators (MLEs) from order $\mathcal{O}\left(n^{-1}\right)$ to $\mathcal{O}\left(n^{-2}\right)$. In addition, there are a biascorrected approach (BCMLE) and a bootstrap approach (BOOT). Various scenarios in Monte Carlo simulations are proceeded to compare the effectiveness of estimators among MLEs, BCMLE, and BOOT methods. As a result, we found that the root mean square error of BCMLE is less than MLEs and BOOT. Similarly, when BCMLE MLEs and BOOT are applied to real datasets, the BSMLE has the smallest standard error.
\end{abstract}

Keywords: Bootstrap bias-correction, Cox-Snell bias-correction, Maximum likelihood estimators, Modified power function distribution, Monte Carlo simulation

\section{Introduction}

The data is often described by the probability distribution function, which is beneficial to predict the possibility of the target random variable. In literature, the bounded variables on $(0,1)$ were often explained by uniform, beta [1], and Kumaraswamy distributions [2]. Recently, in 2017, Ogbonnaya et al. [3] presented alternative distribution to bounded variables on $(0,1)$, namely the extended power distribution. This distribution is derived by extending the single parameter power warping function and can be considered as a more flexible distribution than beta and Kumaraswamy distributions.

In addition to extended power distribution, Okorie et al. [4] also proposed the modified power function (MPF) distribution based on the Marshall-Olkin framework [5]. The cumulative density function (cdf) of the Marshall-Olkin family of distribution is;

$F(x)=1-\frac{\gamma \bar{G}(x)}{1-(1-\gamma) \bar{G}(x)}, \quad-\infty<x<\infty, \gamma>0$,

where $\bar{G}(x)$ is the survival function of a specific distribution. When $\bar{G}(x)$ is the survival function of the 1 parameter power function distribution given by;

$\bar{G}(x)=(1-x)^{\delta}, \quad 0<x<1, \delta>0$,

then the cdf of random variable $X$ becomes;

$F(x)=1-\frac{\gamma(1-x)^{\delta}}{1-(1-\gamma)(1-x)^{\delta}}, \quad 0<x<1, \delta>0, \gamma>0$ 
where $\delta$ and $\gamma$ are the shape parameters. Furthermore, the probability density function (pdf) for the MPF distribution is;

$f(x)=\gamma \delta(1-x)^{\delta-1}\left(1-(1-\gamma)(1-x)^{\delta}\right)^{-2}$.

As demonstrated by Okorie et al. [4], the pdf of the MPF distribution can be increasing, decreasing, unimodal and bathtub. Moreover, the convexity of $f(x)$ was also investigated.

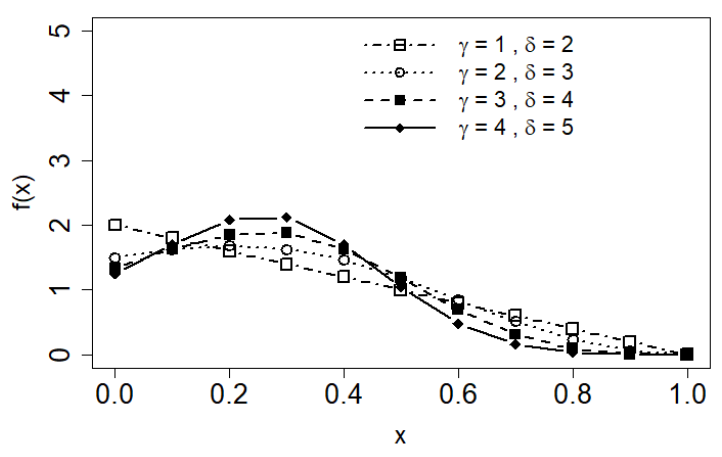

(a)

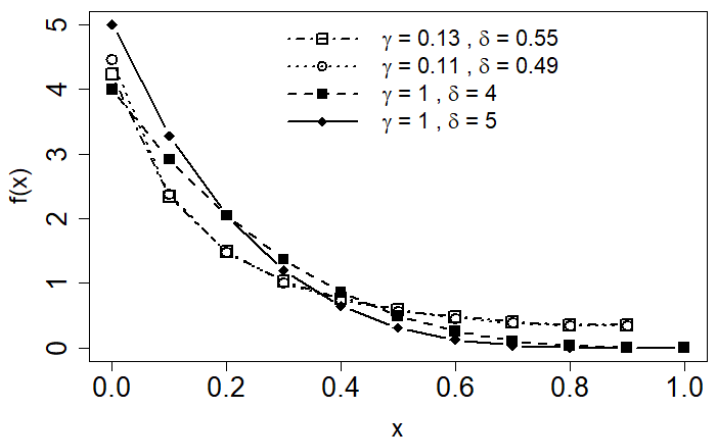

(c)

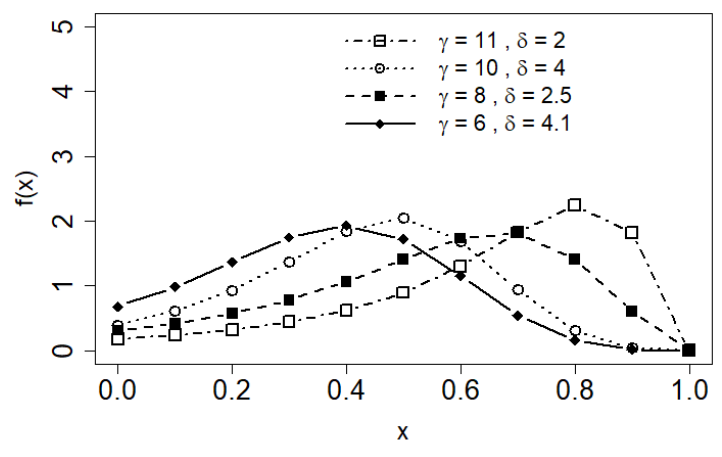

(b)

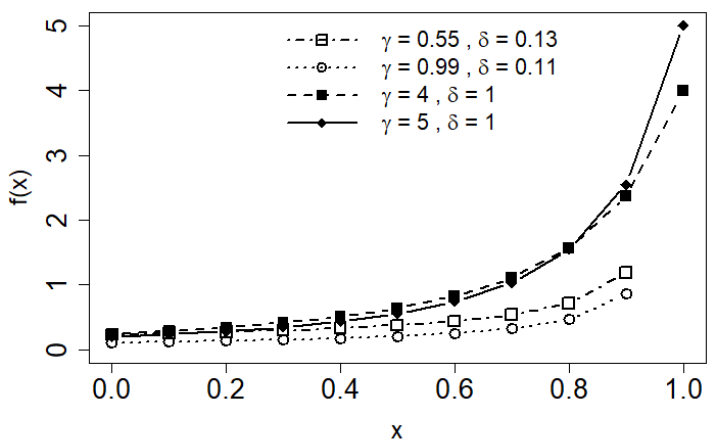

(d)

Figure 1 The shape of the pdf for the MPF distribution when (a) parameter $\gamma=1,2,3,4$ and parameter $\delta=2,3,4,5$ (b) parameter $\gamma=11,10,8,6$ and parameter $\delta=2,4,2.5,4.1$ (c) parameter $\gamma=$ $0.13,0.11,1,1$ and parameter $\delta=0.55,0.49,4,5$ (d) parameter $\gamma=0.55,0.99,4,5$ and parameter $\delta=0.13,0.11,1,1$.

As the MPF distribution was created based on Marshall-Olkin's scheme, this distribution has various shapes, which make it capable of fitting many kinds of real data. The shapes of the MPF distribution are shown in Figure 1. Consequently, the MPF distribution has been investigated on its generalization such as a new generalization of power function distribution [6], the exponentiated generalized power function distribution [7].

The maximum likelihood estimation (MLE) is one of the well-known parameter estimation methods. Frequently, the relative biased with order $\mathcal{O}\left(n^{-1}\right)$ was exit, especially in a small sample size. In literature, many researchers make an attempt to deal with this bias issue for MLE. It is the bias correction method that is applied to compromise MLE estimates bias. The first method is the bias correction initially developed by Cox and Snell [8] in 1968. The second one is the preventive approach proposed by Firth [9] in 1993. However, in this work, we mainly focus on the bias correction method including corrective bias of Cox and Snell [8] and bootstrap approach of Efron [10], respectively. The advantages of bias-corrected MLEs have been investigated for various distributions. For example, Cordeiro et al. [11] and Cribari-Nato et al. [12] have studied and derived estimates with less bias for the beta distribution. The improved point estimation for the Kumaraswamy distribution was presented by Lemonte [13]. Recently, bias-corrected MLEs have been proposed to the Johnson $S_{B}$ distribution [14] and the Lognormal distribution [15], 
respectively. However, for distribution on $(0,1)$, the literature revealed only the work of Mazucheli and Menezes [16] on the unit-gamma distribution, which is related to this estimation aspect. Therefore, bias reduction is essential for delivering effective estimates for distribution on $(0,1)$.

As noted previously, we are driven to produce estimators of the MPF distribution that are approximately unbiased. In section 2, we derive the MLEs for MPF distribution. Then, biased-corrected methodologies such as the corrective and bootstrap methods are explored. Monte Carlo simulations with many case situations are run. Section 4 discusses the root mean square error (RMSE) value resulting from MLE, bias correction MLE, and bootstrapping bias correction. Section 5 illustrates the application with real data.

\section{The maximum likelihood estimation}

For the MPF distribution, the maximum likelihood estimators (MLEs) will be derived for its shape parameters. Let $\mathrm{x}=\left(x_{1}, \ldots, x_{n}\right)^{\prime}$ be a random sample of the MPF distribution with parameter vector $\Theta=(\gamma, \delta)^{\prime}$, the log-likelihood function of $\Theta$ is given by;

$\ell(\Theta)=n \log (\gamma \delta)+(\delta-1) \sum_{i=1}^{n} \log \left(1-x_{i}\right)-2 \sum_{i=1}^{n} \log \left(1-(1-\gamma)\left(1-x_{i}\right)^{\delta}\right)$.

The MLE, $\hat{\Theta}=(\hat{\gamma}, \hat{\delta})^{\prime}$, is obtained from differentiating with respect to $\gamma$ and $\beta$ as follows;

$$
\begin{aligned}
& \frac{\partial \ell(\Theta)}{\partial \gamma}=\frac{n}{\gamma}-2 \sum_{i=1}^{n} \frac{\left(1-x_{i}\right)^{\delta}}{1-(1-\gamma)\left(1-x_{i}\right)^{\delta}} \\
& \frac{\partial \ell(\Theta)}{\partial \delta}=\frac{n}{\delta}+\sum_{i=1}^{n} \log \left(1-x_{i}\right)+2 \sum_{i=1}^{n} \frac{(1-\gamma)\left(1-x_{i}\right)^{\delta} \log \left(1-x_{i}\right)}{1-(1-\gamma)\left(1-x_{i}\right)^{\delta}} .
\end{aligned}
$$

The MLEs can be obtained from setting Eqs. (6) - (7) equal to 0 and solving these equations simultaneously. We implement the numerical method in the optim function of the R language [16] to find the optimal value of the parameters.

\section{The bias-corrected MLEs}

In this section, we perform 2 biased-corrected techniques which can decrease the bias of the MLEs. The first technique is a corrective approach and the other technique is a bootstrap approach.

\section{A corrective approach}

A corrective approach was presented by Cox and Snell [8]. They exhibited the bias of the $s$-th element of the $\operatorname{MLE}=\left(\theta_{1}, \theta_{2}, \ldots, \theta_{p}\right)^{\prime}, \hat{\theta}$, for the sample data with independent, but not necessarily identically distributed, can be written as;

$\operatorname{Bias}\left(\hat{\theta}_{s}\right)=\sum_{i=1}^{p} \sum_{j=1}^{p} \sum_{l=1}^{p} \kappa^{s i} \kappa^{j l}\left(\frac{1}{2} \kappa_{i j l}+\kappa_{i j, l}\right)+O\left(n^{-2}\right)$

where $s=1,2,3, \ldots, p, p$ is unknown parameters, $\kappa^{i j}$ is the $(i, j)$-th element of the inverse of the Fisher information matrix, $\kappa_{i j l}=\mathbb{E}\left(\frac{\partial^{3} \ell(\theta)}{\partial \theta_{i} \partial \theta_{j} \partial \theta_{l}}\right), \kappa_{i j, l}=\mathbb{E}\left(\left(\frac{\partial^{2} \ell(\theta)}{\partial \theta_{i} \partial \theta_{j}}\right)\left(\frac{\partial \ell(\theta)}{\partial \theta_{l}}\right)\right)$ and $\ell(\theta)$ is log-likelihood function. In 1994, Cordeiro and Klein [17] presented general formula in case that the sample data are not identically distributed and still usable for non-independent observations. This formula is;

$\operatorname{Bias}\left(\hat{\theta}_{s}\right)=\sum_{i=1}^{p} \kappa^{s i} \sum_{j=1}^{p} \sum_{l=1}^{p} \kappa^{j l}\left(\kappa_{i j}^{(l)}-\frac{1}{2} \kappa_{i j l}\right)+O\left(n^{-2}\right), \quad s=1,2, \ldots, p$.

where $\kappa_{i j}^{(l)}=\frac{\partial \kappa_{i j}}{\partial \Theta_{l}}$ for $i, j, l=1,2$.

For the bias-corrected MLEs of the MPF distribution, we have $p=2$ with $\Theta=(\gamma, \delta)^{\prime}$. The regularity conditions on the behavior of the log-likelihood function are assumed. The Fisher information matrix of $\Theta$ for the MPF distribution is $K=\left[-\kappa_{i j}\right]$ where $i, j=1,2$; 


$$
\begin{aligned}
& \kappa_{11}=-\frac{n}{\gamma^{2}}+2 \gamma n \sum_{m=0}^{\infty}\left(\frac{\Gamma(m+4)}{\Gamma(m+1) \Gamma(4)} \cdot \frac{(1-\gamma)^{m}}{(m+3)}\right), \\
& \kappa_{12}=\kappa_{21}=\frac{2 \gamma n}{\delta} \sum_{m=0}^{\infty}\left(\frac{\Gamma(m+3)}{\Gamma(m+1) \Gamma(3)}\left(\frac{(1-\gamma)^{m}}{(m+2)^{2}}-\frac{(1-\gamma)^{m+1}}{3(m+3)}\right)\right), \\
& \kappa_{22}=-\frac{n}{\delta^{2}}+\frac{4 \gamma n}{\delta^{2}} \sum_{m=0}^{\infty}\left(\frac{\Gamma(m+3)}{\Gamma(m+1) \Gamma(3)}\left(\frac{(1-\gamma)^{m+1}}{(m+2)^{3}}+\frac{(1-\gamma)^{m+2}}{3(m+3)^{2}}\right)\right) .
\end{aligned}
$$

We derive extensive algebraic of these joint cumulants (Appendix A) $\kappa_{i j l}$ from the higher-order derivatives of the log-likelihood function of $\Theta$, as follows;

$$
\begin{aligned}
& \kappa_{111}=\frac{2 n}{\gamma^{3}}-4 \gamma n \sum_{m=0}^{\infty}\left(\frac{\Gamma(m+5)}{\Gamma(m+1) \Gamma(5)} \cdot \frac{(1-\gamma)^{m}}{(m+4)}\right), \\
& \kappa_{112}=-\frac{4 \gamma n}{\delta} \sum_{m=0}^{\infty}\left(\frac{\Gamma(m+5)}{\Gamma(m+1) \Gamma(5)} \cdot \frac{(1-\gamma)^{m}}{(m+3)^{2}}\right), \\
& \kappa_{122}=-\frac{4 \gamma n}{\delta^{2}} \sum_{m=0}^{\infty}\left(\frac{\Gamma(m+4)}{\Gamma(m+1) \Gamma(4)}\left(\frac{(1-\gamma)^{m}}{(m+2)^{3}}+\frac{(m+4)(1-\gamma)^{m+1}}{2(m+3)^{3}}\right)\right), \\
& \kappa_{222}=\frac{2 n}{\delta^{3}}+\frac{12 \gamma n}{\delta^{3}}\left(\frac{\Gamma(m+4)}{\Gamma(m+1) \Gamma(4)}\left(\frac{(1-\gamma)^{m+1}}{(m+2)^{4}}+\frac{(m+4)(1-\gamma)^{m+2}}{2(m+3)^{4}}\right)\right) .
\end{aligned}
$$

As the higher-order derivatives do not depend on the sample data and thus they are equal to their expectation. Moreover, the matrix form of the bias expression of $\widehat{\Theta}$ for the MPF distribution can be shown as;

$$
\operatorname{Bias}(\hat{\Theta})=K^{-1} A \cdot \operatorname{vec}\left(K^{-1}\right)+O\left(n^{-2}\right),
$$

where $K^{-1}$ is the inverse Fisher information matrix for MPF distribution, a matrix $A=\left[A^{(1)} \mid A^{(2)}\right]$ with $A^{(l)}=\left[a_{i j}^{(l)}\right], a_{i j}^{(l)}=\kappa_{i j}^{(l)}-\frac{1}{2} \kappa_{i j l}$, and $\operatorname{vec}\left(K^{-1}\right)$ is the vector obtained by stacking the column of $K^{-1}$.

To implement the corrective approach, we obtain the element of $A^{(1)}$;

$$
\begin{aligned}
a_{11}^{(1)}= & \frac{n}{\gamma^{3}}+2 n \sum_{m=0}^{\infty}\left(\frac{\Gamma(m+4)}{\Gamma(m+1) \Gamma(4)}\left(\frac{\gamma(1-\gamma)^{m}}{4}-\frac{(m \gamma+\gamma-1)(1-\gamma)^{m-1}}{(m+3)}\right)\right) \\
a_{12}^{(1)}= & a_{21}^{(1)}=-\frac{2 n}{\delta} \sum_{m=0}^{\infty}\left(\frac { \Gamma ( m + 3 ) } { \Gamma ( m + 1 ) \Gamma ( 3 ) } \left(\frac{(m \gamma+\gamma-1)(1-\gamma)^{m-1}}{(m+2)^{2}}\right.\right. \\
& \left.\left.-\frac{(m-4(m+2) \gamma)(1-\gamma)^{m}}{12(m+3)}\right)\right) \\
a_{22}^{(1)}= & \frac{2 n}{3 \delta^{2}} \sum_{m=0}^{\infty}\left(\frac { \Gamma ( m + 3 ) } { \Gamma ( m + 1 ) \Gamma ( 3 ) } \left(\frac{(m-6(m+2) \gamma+9)(1-\gamma)^{m}}{(m+2)^{3}}\right.\right. \\
& \left.\left.+\frac{(m-4(m+3) \gamma+8)(1-\gamma)^{m+1}}{3(m+3)^{2}}\right)\right) .
\end{aligned}
$$

The element of $A^{(2)}$ are; 


$$
\begin{aligned}
a_{11}^{(2)}= & \frac{2 \gamma n}{\delta} \sum_{m=0}^{\infty}\left(\frac{\Gamma(m+5)}{\Gamma(m+1) \Gamma(5)} \cdot \frac{(1-\gamma)^{m}}{(m+3)^{2}}\right), \\
a_{12}^{(2)}=a_{21}^{(2)}= & -\frac{2 \gamma n}{3 \delta^{2}} \sum_{m=0}^{\infty}\left(\frac{\Gamma(m+3)}{\Gamma(m+1) \Gamma(3)}\left(\frac{(3 m+14)(1-\gamma)^{m+1}}{3(m+3)}-\frac{(2 m+3)(1-\gamma)^{m}}{(m+2)^{3}}\right)\right), \\
a_{22}^{(2)}= & \frac{n}{\delta^{3}}-\frac{2 \gamma n}{\delta^{3}} \sum_{m=0}^{\infty}\left(\frac { \Gamma ( m + 3 ) } { \Gamma ( m + 1 ) \Gamma ( 3 ) } \left(\frac{(5 m+11)(1-\gamma)^{m+1}}{(m+2)^{4}}\right.\right. \\
& \left.\left.+\frac{(11 m+36)(1-\gamma)^{m+2}}{6(m+3)^{3}}\right)\right) .
\end{aligned}
$$

Therefore, a bias-corrected MLEs (MLE.BC) of the MPF distribution for $\Theta$ is;

$\hat{\Theta}^{B C M L E}=\left(\begin{array}{l}\hat{\gamma}^{B C M L E} \\ \hat{\delta}^{B C M L E}\end{array}\right)=\left(\begin{array}{l}\hat{\gamma} \\ \hat{\delta}\end{array}\right)-\widehat{K}^{-1} \hat{A} \cdot \operatorname{vec}\left(\widehat{K}^{-1}\right)$,

where $\widehat{K}=\left.K\right|_{\gamma=\widehat{\gamma}, \delta=\widehat{\delta}}$ and $\hat{A}=\left.A\right|_{\gamma=\widehat{\gamma}, \delta=\widehat{\delta}}$. The bias-corrected estimators in Eq. (24) can be computed by using mle.tools package [19] in $\mathrm{R}$ language.

\section{A bootstrap approach}

As the corrective approach requires many algebraic expressions, the bootstrap approach [10] frequently be considered as an alternative method to reduce bias. The bootstrap method can be categorized as a parametric approach by sampling from a fitted parametric model; on the other hand, it becomes a nonparametric approach by sampling with replacement from the original sample. This method has the bootstrap sample as a crucial part. In addition, many works emphasized that the bootstrap method is beneficial to improve the accuracy of the inference [13,20]. Suppose observed data is $\mathrm{x}=$ $\left(x_{1}, x_{2}, \ldots, x_{n}\right)^{\prime}$ and $\hat{\Theta}=(\hat{\gamma}, \hat{\delta})^{\prime}$ is MLE estimates from the MPF distribution. Let $x_{j}^{*}=\left(x_{1 j}^{*}, x_{2 j}^{*}, \ldots, x_{n j}^{*}\right)^{\prime}$ be vector of bootstrap sample, where $j=1,2, \ldots, n$. The bootstrap bias correction method can be obtained by the following steps:

1) Let $j=1$

2) Generate random sample $\mathrm{x}_{j}^{*}=\left(x_{1 j}^{*}, x_{2 j}^{*}, \ldots, x_{n j}^{*}\right)^{\prime}$ from $F_{\widehat{\Theta}}$ with sample size $n$

3) Compute estimates $\Theta_{j}^{*}=\left(\gamma_{j}^{*}, \delta_{j}^{*}\right)^{\prime}$ given $x_{j}^{*}$ by using the MLE method

4) Let $\mathrm{j}=\mathrm{j}+1$. Repeat 2) - 3) until $j=B$

5) Calculate the mean of the bootstrap estimates;

$\bar{\Theta}^{*}=\frac{1}{B} \sum_{j=1}^{B} \Theta_{j}^{*}$.

6) The bootstrap bias-corrected estimator (BOOT) is accomplished by;

$\operatorname{Bias}(\widehat{\Theta})=2 \widehat{\Theta}-\bar{\Theta}^{*}$

(26)

\section{Simulation study}

In this section, we conduct a Monte-Carlo simulation study to compare the performance of the MLEs and their bias-corrected versions obtained by the Cox-Snell methodology and bootstrap method. There are twenty scenarios for parameter values with various sample sizes, $M=1,000$ Monte-Carlo replications, and $B=500$ bootstrap replicates. In addition, simulation study is performed by taking the parameter $\gamma=\{0.2,0.55,2.7,4,6\}$, the parameter $\delta=\{0.8,1.8,7,10\}$, and samples sizes $n=$ $\{15,20,30,40,50\}$. The efficiency of parameter estimation methods is evaluated by the root mean square error (RMSE) criterion. To generate random variate in each scenario, we apply the transformation method with the MPF distribution function. Therefore, random variate is obtained by; 
$X=1-\left(\frac{1-U}{1-U+U \gamma}\right)^{\frac{1}{\delta}}$

where $U$ is the continuous uniform distribution. The results of MLEs, BCMLEs, and BOOT related to parameters $\gamma$ and $\delta$ from the simulation study are reported in Tables $\mathbf{1}$ - $\mathbf{5}$.

From Tables 1 - 5, estimated RMSE of MLE, MLEBC, and BOOT has a tendency to decrease when the sample size is larger. Considering almost all cases, the estimated $\gamma$ and $\delta$ from MLEBC frequency has smaller RMSEs than those from MLE and BOOT. Remarkably, the difference between RMSE of MLEBC and MLE when sample size $n=15$ is larger than those values of difference when sample sizes $n=20,30,40,50$. For example, in the case of $\gamma=0.55$ and $\delta=0.8$, the difference between RMSE of MLEBC and MLE is $|0.5678-1.9983|=1.4305$ when $n=15$, while the difference is $\mid 0.5508-$ $1.1179 \mid=0.5671$ when $n=20$. It indicates that MLEBC estimates usually approach real value, especially in a small sample size.

Table 1 Estimated root mean-squared error for $\gamma$ and $\delta(\gamma=0.2)$.

\begin{tabular}{lccccccc}
\hline $\boldsymbol{\delta}$ & $\mathbf{n}$ & \multicolumn{3}{c}{ RMSE of $\widehat{\gamma}$} & & RMSE of $\widehat{\boldsymbol{\delta}}$ & MLE \\
\cline { 2 - 7 } & 15 & 1.0739 & 0.3731 & 0.7733 & 1.7542 & 1.4059 & 1.4997 \\
\hline 0.8 & 20 & 0.6067 & 0.3031 & 0.4401 & 1.2843 & 1.0350 & 1.0884 \\
& 30 & 0.3389 & 0.2185 & 0.2530 & 0.8545 & 0.7321 & 0.7375 \\
& 40 & 0.2436 & 0.1729 & 0.1855 & 0.6614 & 0.5759 & 0.5735 \\
& 50 & 0.2109 & 0.1636 & 0.1679 & 0.5652 & 0.5223 & 0.5089 \\
\hline 1.8 & 15 & 1.1355 & 0.3890 & 0.8278 & 4.2850 & 3.4994 & 3.7293 \\
& 20 & 0.6559 & 0.3325 & 0.4896 & 2.6866 & 2.2558 & 2.3153 \\
& 30 & 0.4863 & 0.3149 & 0.3771 & 1.8827 & 1.6076 & 1.6246 \\
& 40 & 0.2378 & 0.1725 & 0.1836 & 1.4307 & 1.2675 & 1.2489 \\
& 50 & 0.2030 & 0.1571 & 0.1624 & 1.2862 & 1.1743 & 1.1553 \\
\hline 7 & 15 & 1.0459 & 0.3624 & 0.7664 & 15.4895 & 12.4866 & 13.4916 \\
& 20 & 0.7668 & 0.3718 & 0.5802 & 11.6166 & 9.5510 & 10.1149 \\
& 30 & 1.0196 & 0.6551 & 0.7669 & 22.7297 & 19.4269 & 19.7051 \\
& 40 & 0.2781 & 0.2000 & 0.2180 & 6.1921 & 5.4891 & 5.5104 \\
& 50 & 0.1946 & 0.1516 & 0.1559 & 4.6772 & 4.2777 & 4.2211 \\
\hline 10 & 15 & 0.9759 & 0.3407 & 0.7206 & 21.6269 & 17.2452 & 18.8645 \\
& 20 & 0.6045 & 0.3042 & 0.4474 & 15.8825 & 13.0059 & 13.8165 \\
& 30 & 0.3347 & 0.2169 & 0.2550 & 10.2803 & 8.9339 & 9.0670 \\
& 40 & 0.2420 & 0.1770 & 0.1909 & 8.1217 & 7.2566 & 7.2560 \\
& 50 & 0.2089 & 0.1617 & 0.1706 & 6.8250 & 6.2406 & 6.2217 \\
\hline
\end{tabular}

Table 2 Estimated root mean-squared error for $\gamma$ and $\delta(\gamma=0.55)$.

\begin{tabular}{cccccccc}
\hline $\boldsymbol{\delta}$ & $\mathbf{n}$ & \multicolumn{3}{c}{ RMSE of $\widehat{\boldsymbol{\gamma}}$} & \multicolumn{3}{c}{ RMSE of $\widehat{\boldsymbol{\delta}}$} \\
\cline { 3 - 7 } & & MLE & BCMLE & BOOT & MLE & BCMLE & BOOT \\
\hline 0.8 & 15 & 1.9984 & 0.5678 & 1.4459 & 0.8899 & 0.7267 & 0.7814 \\
& 20 & 1.1179 & 0.5508 & 0.8182 & 0.6592 & 0.5612 & 0.5844 \\
& 30 & 0.6349 & 0.4283 & 0.4890 & 0.4490 & 0.4158 & 0.4141 \\
& 40 & 0.5106 & 0.3807 & 0.4102 & 0.4034 & 0.3762 & 0.3765 \\
& 50 & 0.4510 & 0.3530 & 0.3683 & 0.3516 & 0.3300 & 0.3315 \\
\hline 1.8 & 15 & 1.9252 & 0.5672 & 1.3923 & 1.9074 & 1.5455 & 1.6669 \\
& 20 & 1.1342 & 0.5589 & 0.8334 & 1.4760 & 1.2853 & 1.3256 \\
& 30 & 0.6001 & 0.4007 & 0.4603 & 0.9892 & 0.8985 & 0.9023 \\
& 40 & 0.4758 & 0.3594 & 0.3808 & 0.7926 & 0.7508 & 0.7447 \\
& 50 & 0.4014 & 0.3112 & 0.3225 & 0.7079 & 0.6617 & 0.6600 \\
\hline 7 & 15 & 2.4647 & 0.5559 & 1.8563 & 7.6195 & 6.2687 & 6.7625 \\
& 20 & 0.9998 & 0.5076 & 0.7290 & 5.2018 & 4.5707 & 4.6792
\end{tabular}




\begin{tabular}{cccccccc}
\hline $\boldsymbol{\delta}$ & $\mathbf{n}$ & \multicolumn{3}{c}{ RMSE of $\widehat{\boldsymbol{\gamma}}$} & \multicolumn{3}{c}{ RMSE of $\widehat{\boldsymbol{\delta}}$} \\
\cline { 3 - 8 } & MLE & BCMLE & BOOT & MLE & BCMLE & BOOT \\
\hline & 30 & 0.6635 & 0.4331 & 0.5095 & 3.8899 & 3.5254 & 3.5586 \\
& 40 & 0.4768 & 0.3607 & 0.3847 & 3.1992 & 3.0500 & 3.0336 \\
& 50 & 0.4348 & 0.3404 & 0.3569 & 2.9998 & 2.8145 & 2.8260 \\
\hline 10 & 15 & 2.0114 & 0.5909 & 1.4535 & 10.7889 & 8.9418 & 9.6442 \\
& 20 & 0.9723 & 0.4948 & 0.7162 & 7.7659 & 6.7993 & 6.9817 \\
& 30 & 0.6410 & 0.4204 & 0.4913 & 5.6921 & 5.1444 & 5.2111 \\
& 40 & 0.4816 & 0.3612 & 0.3831 & 4.6055 & 4.3691 & 4.3365 \\
& 50 & 0.4224 & 0.3283 & 0.3445 & 4.0582 & 3.7978 & 3.8149 \\
\hline
\end{tabular}

Table 3 Estimated root mean-squared error for $\gamma$ and $\delta(\gamma=2.7)$.

\begin{tabular}{|c|c|c|c|c|c|c|c|}
\hline \multirow[t]{2}{*}{$\delta$} & \multirow[t]{2}{*}{$\mathbf{n}$} & \multicolumn{3}{|c|}{ RMSE of $\widehat{\gamma}$} & \multicolumn{3}{|c|}{ RMSE of $\widehat{\delta}$} \\
\hline & & MLE & BCMLE & BOOT & MLE & BCMLE & BOOT \\
\hline \multirow[t]{5}{*}{0.8} & 15 & 7.1505 & 1.5329 & 5.0891 & 0.3901 & 0.3403 & 0.3685 \\
\hline & 20 & 4.9987 & 1.8897 & 3.6203 & 0.3155 & 0.2849 & 0.3006 \\
\hline & 30 & 2.9258 & 1.7062 & 2.2378 & 0.2403 & 0.2205 & 0.2287 \\
\hline & 40 & 2.1436 & 1.4564 & 1.6525 & 0.2035 & 0.1917 & 0.1959 \\
\hline & 50 & 1.7616 & 1.3177 & 1.4168 & 0.1774 & 0.1682 & 0.1713 \\
\hline \multirow[t]{5}{*}{1.8} & 15 & 7.2391 & 1.5994 & 5.1795 & 0.8745 & 0.7778 & 0.8347 \\
\hline & 20 & 7.8399 & 1.9789 & 5.6968 & 0.7353 & 0.6759 & 0.7103 \\
\hline & 30 & 2.7877 & 1.6891 & 2.1330 & 0.5433 & 0.5138 & 0.5272 \\
\hline & 40 & 2.0904 & 1.5071 & 1.6814 & 0.4437 & 0.4322 & 0.4380 \\
\hline & 50 & 1.8800 & 1.4358 & 1.5426 & 0.4198 & 0.4077 & 0.4130 \\
\hline \multirow[t]{5}{*}{7} & 15 & 7.2679 & 1.5916 & 5.2030 & 3.4233 & 3.0581 & 3.2703 \\
\hline & 20 & 5.2167 & 1.9112 & 3.9074 & 2.7861 & 2.5766 & 2.6888 \\
\hline & 30 & 3.0316 & 1.8236 & 2.3345 & 3.1931 & 3.0246 & 3.1108 \\
\hline & 40 & 2.2976 & 1.6192 & 1.8227 & 2.5991 & 2.4959 & 2.5449 \\
\hline & 50 & 1.7683 & 1.3315 & 1.4373 & 2.3150 & 2.2252 & 2.2668 \\
\hline \multirow[t]{5}{*}{10} & 15 & 6.7555 & 1.6030 & 4.8383 & 4.6949 & 4.2297 & 4.5125 \\
\hline & 20 & 4.1848 & 1.7741 & 3.0706 & 3.9013 & 3.6335 & 3.7938 \\
\hline & 30 & 3.0316 & 1.8236 & 2.3345 & 3.1931 & 3.0246 & 3.1108 \\
\hline & 40 & 2.2976 & 1.6192 & 1.8227 & 2.5991 & 2.4959 & 2.5449 \\
\hline & 50 & 1.7683 & 1.3315 & 1.4373 & 2.3150 & 2.2252 & 2.2668 \\
\hline
\end{tabular}

Table 4 Estimated root mean-squared error for $\gamma$ and $\delta(\gamma=4)$.

\begin{tabular}{lccccccc}
\hline $\boldsymbol{\delta}$ & $\mathbf{n}$ & \multicolumn{3}{c}{ RMSE of $\hat{\boldsymbol{\gamma}}$} & & RMSE of $\widehat{\boldsymbol{\delta}}$ & BOE \\
\cline { 2 - 7 } & 15 & MLE & BCMLE & BOOT & BCMLE & BOOT \\
\hline 0.8 & 20 & 7.4956 & 2.2054 & 6.8862 & 0.3551 & 0.3060 & 0.3383 \\
& 30 & 4.6884 & 2.6447 & 5.5220 & 0.2765 & 0.2515 & 0.2670 \\
& 40 & 3.3453 & 2.2409 & 2.6083 & 0.2254 & 0.2103 & 0.2189 \\
& 50 & 2.6383 & 1.9760 & 2.1529 & 0.1808 & 0.1700 & 0.1746 \\
& 15 & 9.3153 & 2.2615 & 6.6028 & 0.7527 & 0.6682 & 0.7241 \\
\hline 1.8 & 20 & 7.6923 & 2.5761 & 5.6219 & 0.6695 & 0.6052 & 0.6457 \\
& 30 & 4.3101 & 2.5115 & 3.2664 & 0.5200 & 0.4917 & 0.5070 \\
& 40 & 3.2203 & 2.1774 & 2.5362 & 0.4208 & 0.4030 & 0.4118 \\
& 50 & 2.3856 & 1.8551 & 1.9884 & 0.3351 & 0.3342 & 0.3367 \\
\hline 7 & 15 & 8.8973 & 2.2998 & 6.2697 & 3.0577 & 2.7264 & 2.9449 \\
& 20 & 6.8580 & 2.4314 & 4.9792 & 2.4559 & 2.2459 & 2.3786 \\
& 30 & 4.5339 & 2.5969 & 3.4592 & 1.9482 & 1.8682 & 1.9232 \\
& 40 & 3.4623 & 2.3465 & 2.7307 & 1.6634 & 1.5961 & 1.6332 \\
& 50 & 2.7860 & 2.0993 & 2.3085 & 1.3948 & 1.3633 & 1.3845 \\
\hline 10 & 15 & 9.2144 & 2.3169 & 6.6040 & 4.1945 & 3.7838 & 4.0670 \\
& 20 & 7.8425 & 2.6836 & 5.7453 & 3.5484 & 3.3159 & 3.4745 \\
& 30 & 4.6646 & 2.6435 & 3.5513 & 2.7750 & 2.6562 & 2.7317
\end{tabular}




\begin{tabular}{cccccccc}
\hline $\boldsymbol{\delta}$ & $\mathbf{n}$ & \multicolumn{3}{c}{ RMSE of $\hat{\boldsymbol{\gamma}}$} & \multicolumn{3}{c}{ RMSE of $\widehat{\boldsymbol{\delta}}$} \\
\cline { 3 - 7 } & MLE & BCMLE & BOOT & MLE & BCMLE & BOOT \\
\hline & 30 & 3.6337 & 2.4470 & 2.8634 & 2.3733 & 2.2778 & 2.3279 \\
& 50 & 2.5761 & 1.9747 & 2.1209 & 1.9890 & 1.9499 & 1.9769 \\
\hline
\end{tabular}

Table 5 Estimated root mean-squared error for $\gamma$ and $\delta(\gamma=6)$.

\begin{tabular}{|c|c|c|c|c|c|c|c|}
\hline \multirow[t]{2}{*}{$\delta$} & \multirow[t]{2}{*}{$\mathbf{n}$} & \multicolumn{3}{|c|}{ RMSE of $\gamma$} & \multicolumn{3}{|c|}{ RMSE of $\delta$} \\
\hline & & MLE & BCMLE & BOOT & MLE & BCMLE & BOOT \\
\hline \multirow[t]{5}{*}{0.8} & 15 & 11.5174 & 3.5718 & 8.0291 & 0.2831 & 0.2446 & 0.2713 \\
\hline & 20 & 14.0855 & 3.6864 & 10.4129 & 0.2678 & 0.2425 & 0.2616 \\
\hline & 30 & 8.4452 & 4.2098 & 6.3853 & 0.2051 & 0.1905 & 0.1993 \\
\hline & 40 & 6.3015 & 3.9789 & 4.8932 & 0.1732 & 0.1641 & 0.1693 \\
\hline & 50 & 4.8002 & 3.4470 & 3.9016 & 0.1564 & 0.1502 & 0.1534 \\
\hline \multirow[t]{5}{*}{1.8} & 15 & 12.0611 & 3.6812 & 8.4067 & 0.6494 & 0.5918 & 0.6360 \\
\hline & 20 & 12.4201 & 3.4280 & 9.0117 & 0.5690 & 0.5281 & 0.5590 \\
\hline & 30 & 8.3376 & 4.2441 & 6.4172 & 0.4672 & 0.4438 & 0.4607 \\
\hline & 40 & 5.0827 & 3.3658 & 3.9682 & 0.3773 & 0.3652 & 0.3736 \\
\hline & 50 & 4.2541 & 3.0534 & 3.4121 & 0.3422 & 0.3295 & 0.3372 \\
\hline \multirow[t]{5}{*}{7} & 15 & 11.8182 & 3.7685 & 8.3856 & 2.5018 & 2.3313 & 2.4728 \\
\hline & 20 & 14.0400 & 3.5476 & 10.3613 & 2.3035 & 2.1067 & 2.2583 \\
\hline & 30 & 6.9026 & 3.7280 & 5.2642 & 1.7834 & 1.7078 & 1.7658 \\
\hline & 40 & 5.1018 & 3.3697 & 4.0303 & 1.5180 & 1.4629 & 1.5009 \\
\hline & 50 & 4.2324 & 3.0620 & 3.4402 & 1.3415 & 1.2991 & 1.3266 \\
\hline \multirow[t]{5}{*}{10} & 15 & 10.7291 & 3.6866 & 7.4374 & 3.5154 & 3.2199 & 3.4364 \\
\hline & 20 & 11.4460 & 3.3598 & 8.2918 & 3.1858 & 2.9232 & 3.1171 \\
\hline & 30 & 8.3182 & 3.9709 & 6.3094 & 2.4810 & 2.3442 & 2.4393 \\
\hline & 40 & 5.4137 & 3.5771 & 4.2706 & 2.1262 & 2.0572 & 2.1038 \\
\hline & 50 & 4.7126 & 3.3790 & 3.7891 & 1.9354 & 1.8691 & 1.9144 \\
\hline
\end{tabular}

\section{Applications}

To exemplify the usefulness of bias correction parameter estimation, we illustrate the fitting results of the MPF distribution to 2 real datasets. In addition, 2 real datasets are rock sample data and total milk production data. The results of MLE and MLEBC methods of the MPF distribution are shown including estimates and corresponding standard error. Then estimates with the least standard error of the MP distribution are compared to the Beta and Kumwaraswamy distributions based on negative log-likelihood values.

Table 6 Total milk production data.

\begin{tabular}{rrrrrrrrrr}
\hline 0.4365 & 0.8781 & 0.2356 & 0.0671 & 0.535 & 0.4371 & 0.4823 & 0.5529 & 0.6750 & 0.3945 \\
0.8492 & 0.2160 & 0.3413 & 0.4111 & 0.4049 & 0.5941 & 0.4260 & 0.4990 & 0.6012 & 0.2361 \\
0.4151 & 0.3383 & 0.5912 & 0.4530 & 0.5113 & 0.4553 & 0.8147 & 0.6707 & 0.4332 & 0.5349 \\
0.5553 & 0.6174 & 0.5140 & 0.6058 & 0.1525 & 0.4800 & 0.6789 & 0.6114 & 0.5744 & 0.3891 \\
0.5447 & 0.4470 & 0.3627 & 0.6220 & 0.0854 & 0.3751 & 0.5878 & 0.6860 & 0.6907 & 0.6891 \\
0.5483 & 0.5707 & 0.4576 & 0.3480 & 0.5481 & 0.4752 & 0.4143 & 0.5285 & 0.3906 & 0.5629 \\
0.3821 & 0.1546 & 0.4741 & 0.0609 & 0.7471 & 0.5770 & 0.6927 & 0.7131 & 0.3259 & 0.4564 \\
0.1131 & 0.3134 & 0.5627 & 0.5232 & 0.4438 & 0.4675 & 0.4694 & 0.4517 & 0.3598 & 0.6488 \\
0.2605 & 0.5394 & 0.7261 & 0.5853 & 0.2303 & 0.7804 & 0.7290 & 0.3175 & 0.5150 & 0.6465 \\
0.4612 & 0.6844 & 0.3635 & 0.2681 & 0.7629 & 0.2747 & 0.6196 & 0.1479 & 0.3323 & 0.6768 \\
0.7687 & 0.3406 & 0.0168 & 0.1167 & 0.0776 & 0.0650 & 0.3188 & & & \\
\hline
\end{tabular}


Table 7 The estimates and standard error of the MPF distribution for the first dataset.

\begin{tabular}{ccccc}
\hline \multirow{2}{*}{ Results } & \multicolumn{2}{c}{ MLE } & \multicolumn{2}{c}{ BCMLE } \\
\cline { 2 - 5 } & $\widehat{\boldsymbol{\gamma}}$ & $\widehat{\boldsymbol{\delta}}$ & $\widehat{\boldsymbol{\gamma}}$ & $\widehat{\boldsymbol{\delta}}$ \\
\hline Estimates & 20.0280 & 3.8389 & 8.4155 & 3.5617 \\
SE & 17.7222 & 0.9028 & 6.6064 & 0.9324 \\
\hline
\end{tabular}

Table 8 The fitting results of the MPF, Beta, and Kumwaraswamy distributions for the first dataset.

\begin{tabular}{cccc}
\hline Model & Estimates & SE & $-\mathbf{L L}$ \\
\hline MPF & $\hat{\gamma}=8.4155$ & 6.6064 & -4.79 \\
& $\hat{\delta}=3.5617$ & 0.9324 & \\
Beta & $\hat{\alpha}=1.7946$ & 0.5361 & -2.10 \\
& $\hat{\beta}=1.9313$ & 0.5823 & \\
Kumwaraswamy & $\hat{\alpha}=1.8584$ & 0.4948 & -2.48 \\
& $\hat{\beta}=2.3165$ & 0.8857 & \\
\hline
\end{tabular}

The first dataset is the proportion of total milk production in the first birth of 107 cows from the SINDI race. These cows are property of the Carnaúba farm which belongs to the Agropecuária Manoel Dantas Ltda (AMDA), located in Taperoá City, Paraíba (Brazil). Total milk production data are present in Table 6.

As the sample size of this dataset is large, we take a random sample of size 20 without replacement from this data set to emphasize the result on small sample sizes. The fitting results of the MPF distribution are illustrated in Table 7. As the standard error of MLEBC is the smallest, its corresponding estimates can be selected as the proper values for the MPF parameter estimation. Based on the negative log-likelihood valuesin Table 8 and the empirical cumulative distribution compared with candidate theoretical distribution functions in Figure 2, we can conclude that the MPF distribution is recommended for fitting this dataset.

Table 9 Rock sample data.

\begin{tabular}{llllllllll}
\hline 0.0903 & 0.1486 & 0.1833 & 0.1171 & 0.1224 & 0.1670 & 0.1897 & 0.1641 & 0.2037 & 0.1624 \\
0.1481 & 0.2286 & 0.2316 & 0.1726 & 0.1535 & 0.2043 & 0.2627 & 0.2001 & 0.1448 & 0.1139 \\
0.2401 & 0.1619 & 0.2809 & 0.1795 & 0.1918 & 0.1331 & 0.2252 & 0.3413 & 0.3116 & 0.2760 \\
0.3266 & 0.1542 & 0.2760 & 0.1770 & 0.4387 & 0.1636 & 0.2538 & 0.3286 & 0.2301 & 0.4641 \\
0.2007 & 0.2627 & 0.1825 & 0.2004 & 0.1509 & 0.2910 & 0.1977 & 0.4205 & & \\
\hline
\end{tabular}

Table 10 The estimates and standard error of the MPF distribution for the second dataset.

\begin{tabular}{crrrr}
\hline \multirow{2}{*}{ Results } & \multicolumn{2}{c}{ MLE } & \multicolumn{2}{c}{ BCMLE } \\
\cline { 2 - 5 } & $\widehat{\gamma}$ & \multicolumn{1}{c}{$\widehat{\boldsymbol{\delta}}$} & \multicolumn{1}{c}{$\widehat{\boldsymbol{\gamma}}$} & \multicolumn{1}{c}{$\widehat{\boldsymbol{\delta}}$} \\
\hline Estimates & 40.2707 & 15.8148 & 29.0435 & 15.3729 \\
SE & 23.1414 & 2.2399 & 16.5641 & 2.2026 \\
\hline
\end{tabular}

Table 11 The fitting results of the MPF, Beta, and Kumwaraswamy distributions for the second dataset.

\begin{tabular}{cccc}
\hline Model & Estimates & SE & -LL \\
\hline MPF & $\hat{\gamma}=29.0435$ & 16.5641 & -51.1445 \\
& $\hat{\delta}=15.3729$ & 2.2026 & \\
Beta & $\hat{\alpha}=5.9430$ & 1.1816 & -55.6002 \\
& $\hat{\beta}=21.2120$ & 4.3482 & \\
Kumwaraswamy & $\hat{\alpha}=2.7190$ & 0.2934 & -52.4915 \\
& $\hat{\beta}=44.6692$ & 17.5711 & \\
\hline
\end{tabular}


For the second dataset, it related to measurements on petroleum rock samples [21]. In the experiment, the researcher used 48 rock samples from a petroleum reservoir. Then, the permeability of rocks was measured including the total area of pores, the total perimeter of pores, and shape [21]. We then analyze the shape perimeter by squared (area) variable showed in Table 9.

The estimates and standard error of the MPF distribution for the second dataset are presented in Table 10 By considering standard error, we can choose estimates of the BCMLE method for describing rock sample data. Then, the Beta and Kumwaraswamy distributions are also applied to this dataset, where the estimates, standard error, and negative log-likelihood values are included in Table 11. Based on the results and the empirical cumulative distribution compared with the 3 theoretical distribution functions in Figure 2, it suggests that the MPF distribution can be considered as an alternative to the Beta and Kumwaraswamy distribution.

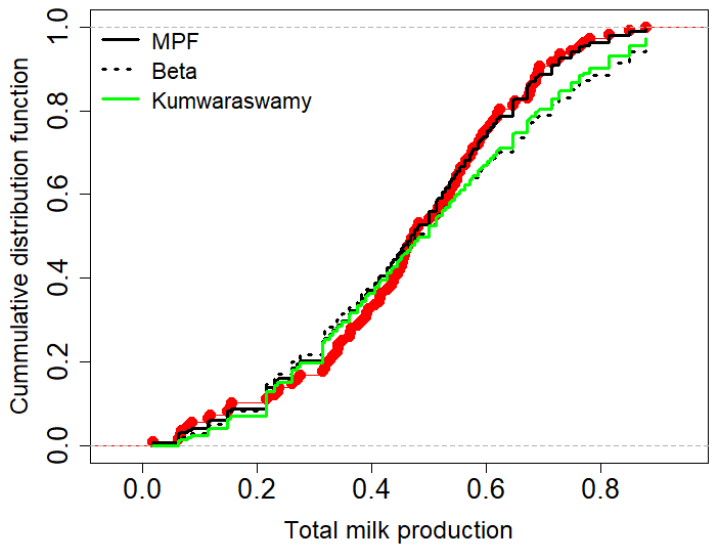

(a)

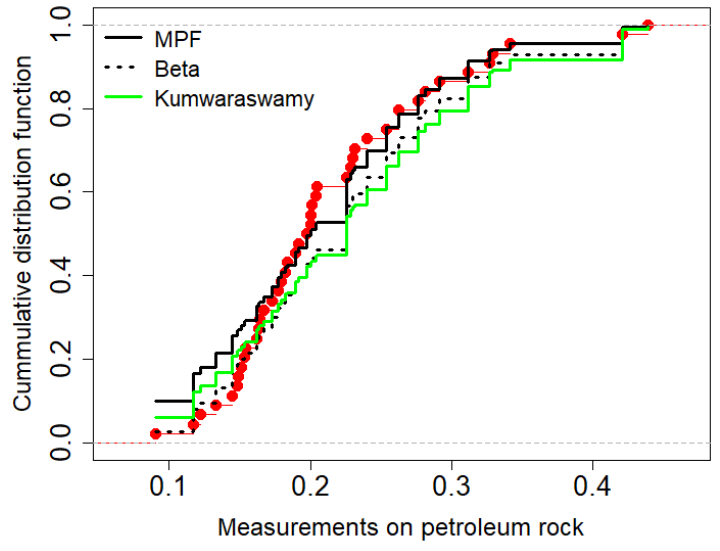

(b)

Figure 2 The empirical cumulative distribution compared to theoretical cumulative distributions associated with the MPF, Beta, and Kumwaraswamy distributions for (a) the total milk data (b) the rock sample data.

\section{Conclusions}

The estimation procedure is a critical component of data analysis. We focused primarily on the maximum likelihood technique in this study. The corrective bias procedure was useful as it reduced the bias of MLEs from order $\mathcal{O}\left(n^{-1}\right)$ to order $\mathcal{O}\left(n^{-2}\right)$. However, corrective bias procedure was rarely investigated on bounded distribution on $(0,1)$ in literature. As MPF distribution has been recently proposed, it also shows flexible shapes of distribution and can be considered to the beta and the kumwaraswamy distributions. Therefore, we proposed biased-corrected MLEs to the MPF parameters, including the corrective (BCMLE) and bootstrap (BOOT) methods. In the simulation study, various cases based on parameter values and sample sizes were conducted to compare the RMSE of MLE, BCMLE, and BOOT estimates. Therefore, we can conclude that BCMLE estimates approach to real values of parameters more than others. Moreover, the corrective MLE of the MPF parameters were also applied to real data. By comparing negative log-likelihood values, the MPF distribution outperformed the Beta and Kumwaraswamy distributions in a small sample size.

\section{Acknowledgement}

The first author was gained financial support from the Fund at Walailak University, Thailand (WU_IRG61_21). 


\section{References}

[1] NL Johnson, S Kotz and N Balakrishnan. Continuous univariate distributions. Vol. III. Wiley, New York, 1995.

[2] MC Jones. Kumaraswamy's distribution: A beta-type distribution with some tractability advantages. Stat. Meth. 2009; 6, 70-81.

[3] CE Ogbonnaya, SP Preston and ATA Wood. The extended power distribution: A new distribution on $(0,1)$, Available at: https://arxiv.org/abs/1711.02774, accessed January 2021.

[4] IE Okorie, AC Akpanta, J Ohakwe and DC Chikezie. The modified power function distribution. Cogent Math. 2017; 4, 1319592.

[5] AW Marshall and I Olkin. A new method for adding a parameter to a family of distributions with application to the exponential and Weibull families. Biometrika 1997; 84, 641-52.

[6] AS Hassan and SG Nassr. A new generalization of power function distribution: Properties and estimation based on censored samples. Thailand Stat. 2020; 18, 215-34.

[7] A Zaka, AS Akhter and R Jabeen. The exponentiated generalized Power function distribution: Theory and real life applications. Adv. Appl. Stat. 2020; 61, 33-63.

[8] DR Cox and EJ Snell. A general definition of residuals. J. Royal Stat. Soc. B 1968; 30, 248-75.

[9] D Firth. Bias reduction of maximum likelihood estimates. Biometrika 1993; 80, 27-38.

[10] B Efron. The Jackknife, the Bootstrap and other resampling plans. Society for Industrial and Applied Mathematics, Philadelphia, 1982.

[11] GM Cordeiro, ECD Rocha, JGCD Rocha and F Cribari-Neto.Bias-corrected maximum likelihood estimation for the beta distribution. J. Stat. Comput. Simulat. 1997; 58, 21-35.

[12] F Cribari-Neto and KLP Vasconcellos. Nearly unbiased maximum likelihood estimation for the Beta distribution. J. Stat. Comput. Simulat. 2002; 72, 107-18.

[13] AJ Lemonte. Improved point estimation for the Kumaraswamy distribution. J. Stat. Comput. Simulat. 2011; 81, 1971-82.

[14] AFB Menezes and J Mazucheli. Improved maximum likelihood estimators for the parameters of the Johnson $\mathrm{S}_{\mathrm{B}}$ distribution. Comm. Stat. Simulat. Comput. 2020; 49, 1511-26.

[15] S Wang and W Gui. Corrected maximum likelihood estimations of the lognormal distribution parameters. Symmetry 2020; 12, 968.

[16] J Mazucheli and AFB Menezes. Improved maximum likelihood estimators for the parameters of the unit-Gamma distribution. Comm. Stat. Theor. Meth. 2017; 47, 3767-78.

[17] GM Cordeiro and R Klein. Bias correction in ARMA models. Stat. Prob. Lett. 1994; 19, $169-76$.

[18] R Core Team. R: A language and environment for statistical computing. R Foundation for Statistical Computing, Vienna, 2020.

[19] J Mazucheli. Mle.tools: Expected/observed fisher information and bias-corrected maximum likelihood estimate(s). R Package version 1.0.0, 2017.

[20] JA Mazucheli, FB Menezes and S Dey. Bias-corrected maximum likelihood estimators of the parameters of the inverse Weibull distribution. Comm. Stat. Simulat. Comput. 2019; 48, 2046-55.

[21] GM Cordeiro and RDS Brito. The beta power distribution. Braz. J. Prob. Stat. 2012; 26, 88-112. 


\section{Appendices}

Appendix A. mathematical derivations of the joint cumulants

To obtain the bias-corrected MLEs for the MPF distribution, we can derive closed-form obviousness of the joint cumulants. These are calculated expected higher-order derivatives of the loglikelihood function of the MPF distribution. Also, we applied the expansion that $(1-q)^{-p}=$ $\sum_{m=0}^{\infty}\left(q^{m} \Gamma(m+p)\right) /(\Gamma(m+1) \Gamma(p))$ where $0 \leq q<1$ for convenience in the calculation. The joint cumulants for the MPF distribution are showed as follows;

$$
\begin{aligned}
\kappa_{11} & =\mathbb{E}\left(\frac{\partial^{2} \ell(\Theta)}{\partial \gamma^{2}}\right), \\
& =\mathbb{E}\left(-\frac{n}{\gamma^{2}}+2 \sum_{i=1}^{n} \frac{\left(1-x_{i}\right)^{2 \delta}}{\left(1-(1-\gamma)\left(1-x_{i}\right)^{\delta}\right)^{2}}\right), \\
& =-\frac{n}{\gamma^{2}}+2 \sum_{i=1}^{n}\left(\int_{0}^{1} \frac{\left(1-x_{i}\right)^{2 \delta}}{\left(1-(1-\gamma)\left(1-x_{i}\right)^{\delta}\right)^{2}} \cdot \frac{\gamma \delta\left(1-x_{i}\right)^{\delta-1}}{\left(1-(1-\gamma)\left(1-x_{i}\right)^{\delta}\right)^{2}} d x_{i}\right), \\
& =-\frac{n}{\gamma^{2}}+2 \gamma \delta \sum_{i=1}^{n}\left(\int_{0}^{1} \frac{\left(1-x_{i}\right)^{3 \delta-1}}{\left(1-(1-\gamma)\left(1-x_{i}\right)^{\delta}\right)^{4}} d x_{i}\right), \\
& =-\frac{n}{\gamma^{2}}+2 \gamma \delta \sum_{i=1}^{n} \sum_{m=0}^{\infty}\left(\frac{\Gamma(m+4)}{\Gamma(m+1) \Gamma(4)} \cdot(1-\gamma)^{m} \int_{0}^{1}\left(1-x_{i}\right)^{\delta(m+3)-1} d x_{i}\right) \\
& =-\frac{n}{\gamma^{2}}+2 \gamma \delta n \sum_{m=0}^{\infty}\left(\frac{\Gamma(m+4)}{\Gamma(m+1) \Gamma(4)} \cdot \frac{(1-\gamma)^{m}}{(m+3)}\right)(\text { Equation }(10)),
\end{aligned}
$$




$$
\begin{aligned}
\kappa_{12}= & \mathbb{E}\left(\frac{\partial^{2} \ell(\Theta)}{\partial \gamma \partial \delta}\right), \\
= & \mathbb{E}\left(-2 \sum_{i=1}^{n}\left(\frac{\left(1-x_{i}\right)^{\delta} \log \left(1-x_{i}\right)}{1-(1-\gamma)\left(1-x_{i}\right)^{\delta}}+\frac{(1-\gamma)\left(1-x_{i}\right)^{2 \delta} \log \left(1-x_{i}\right)}{\left(1-(1-\gamma)\left(1-x_{i}\right)^{\delta}\right)^{2}}\right)\right), \\
= & -2 \sum_{i=1}^{n}\left(\int_{0}^{1} \frac{\left(1-x_{i}\right)^{\delta} \log \left(1-x_{i}\right)}{1-(1-\gamma)\left(1-x_{i}\right)^{\delta}} \cdot \frac{\gamma \delta\left(1-x_{i}\right)^{\delta-1}}{\left(1-(1-\gamma)\left(1-x_{i}\right)^{\delta}\right)^{2}} d x_{i}\right. \\
& \left.+\int_{0}^{1} \frac{(1-\gamma)\left(1-x_{i}\right)^{2 \delta} \log \left(1-x_{i}\right)}{\left(1-(1-\gamma)\left(1-x_{i}\right)^{\delta}\right)^{2}} \cdot \frac{\gamma \delta\left(1-x_{i}\right)^{\delta-1}}{\left(1-(1-\gamma)\left(1-x_{i}\right)^{\delta}\right)^{2}} d x_{i}\right), \\
= & -2 \sum_{i=1}^{n}\left(\int_{0}^{1} \frac{\gamma \delta\left(1-x_{i}\right)^{2 \delta-1} \log \left(1-x_{i}\right)}{\left(1-(1-\gamma)\left(1-x_{i}\right)^{\delta}\right)^{3}} d x_{i}+\int_{0}^{1} \frac{\gamma \delta(1-\gamma)\left(1-x_{i}\right)^{3 \delta-1} \log \left(1-x_{i}\right)}{\left(1-(1-\gamma)\left(1-x_{i}\right)^{\delta}\right)^{4}} d x_{i}\right), \\
= & -2 \gamma \delta \sum_{i=1}^{n} \sum_{m=0}^{\infty}\left(\frac{\Gamma(m+3)}{\Gamma(m+1) \Gamma(3)} \cdot(1-\gamma)^{m} \int_{0}^{1}\left(1-x_{i}\right)^{\delta(m+2)-1} \log \left(1-x_{i}\right) d x_{i}\right) \\
& +2 \gamma \delta(1-\gamma) \sum_{i=1}^{n} \sum_{m=0}^{\infty}\left(\frac{\Gamma(m+4)}{\Gamma(m+1) \Gamma(4)} \cdot(1-\gamma)^{m} \int_{0}^{1}\left(1-x_{i}\right)^{\delta(m+3)-1} \log \left(1-x_{i}\right) d x_{i}\right), \\
= & \frac{2 \gamma n}{\delta} \sum_{m=0}^{\infty}\left(\frac{\Gamma(m+3)}{\Gamma(m+1) \Gamma(3)}\left(\frac{(1-\gamma)^{m}}{(m+2)^{2}}-\frac{(1-\gamma)^{m+1}}{3(m+3)}\right)\right)(\text { Equation }(11)),
\end{aligned}
$$




$$
\begin{aligned}
& \kappa_{22}=\mathbb{E}\left(\frac{\partial^{2} \ell(\Theta)}{\partial \delta^{2}}\right), \\
& =\mathbb{E}\left(-\frac{n}{\delta^{2}}+2 \sum_{i=1}^{n}\left(\frac{(1-\gamma)\left(1-x_{i}\right)^{\delta} \log ^{2}\left(1-x_{i}\right)}{1-(1-\gamma)\left(1-x_{i}\right)^{\delta}}+\frac{(1-\gamma)^{2}\left(1-x_{i}\right)^{2 \delta} \log ^{2}\left(1-x_{i}\right)}{\left(1-(1-\gamma)\left(1-x_{i}\right)^{\delta}\right)^{2}}\right)\right) \text {, } \\
& =-\frac{n}{\delta^{2}}+2 \sum_{i=1}^{n}\left(\int_{0}^{1} \frac{(1-\gamma)\left(1-x_{i}\right)^{\delta} \log ^{2}\left(1-x_{i}\right)}{1-(1-\gamma)\left(1-x_{i}\right)^{\delta}} \cdot \frac{\gamma \delta\left(1-x_{i}\right)^{\delta-1}}{\left(1-(1-\gamma)\left(1-x_{i}\right)^{\delta}\right)^{2}} d x_{i}\right. \\
& \left.+\int_{0}^{1} \frac{(1-\gamma)^{2}\left(1-x_{i}\right)^{2 \delta} \log ^{2}\left(1-x_{i}\right)}{\left(1-(1-\gamma)\left(1-x_{i}\right)^{\delta}\right)^{2}} \cdot \frac{\gamma \delta\left(1-x_{i}\right)^{\delta-1}}{\left(1-(1-\gamma)\left(1-x_{i}\right)^{\delta}\right)^{2}} d x_{i}\right) \\
& =-\frac{n}{\delta^{2}}+2 \sum_{i=1}^{n}\left(\int_{0}^{1} \frac{\gamma \delta(1-\gamma)\left(1-x_{i}\right)^{2 \delta-1} \log ^{2}\left(1-x_{i}\right)}{\left(1-(1-\gamma)\left(1-x_{i}\right)^{\delta}\right)^{3}} d x_{i}\right. \\
& \left.+\int_{0}^{1} \frac{\gamma \delta(1-\gamma)^{2}\left(1-x_{i}\right)^{3 \delta-1} \log ^{2}\left(1-x_{i}\right)}{\left(1-(1-\gamma)\left(1-x_{i}\right)^{\delta}\right)^{4}} d x_{i}\right) \\
& =-\frac{n}{\delta^{2}}+2 \gamma \delta(1-\gamma) \sum_{i=1}^{n} \sum_{m=0}^{\infty}\left(\frac{\Gamma(m+3)}{\Gamma(m+1) \Gamma(3)} \cdot(1-\gamma)^{m} \int_{0}^{1}\left(1-x_{i}\right)^{\delta(m+2)-1} \log ^{2}\left(1-x_{i}\right) d x_{i}\right) \text {, } \\
& +2 \gamma \delta(1-\gamma)^{2} \sum_{i=1}^{n} \sum_{m=0}^{\infty}\left(\frac{\Gamma(m+4)}{\Gamma(m+1) \Gamma(4)} \cdot(1-\gamma)^{m} \int_{0}^{1}\left(1-x_{i}\right)^{\delta(m+3)-1} \log ^{2}\left(1-x_{i}\right) d x_{i}\right) \\
& =-\frac{n}{\delta^{2}}+\frac{4 \gamma n}{\delta^{2}} \sum_{m=0}^{\infty}\left(\frac{\Gamma(m+3)}{\Gamma(m+1) \Gamma(3)}\left(\frac{(1-\gamma)^{m+1}}{(m+2)^{3}}+\frac{(1-\gamma)^{m+2}}{3(m+3)^{2}}\right)\right)(\text { Equation }(12)) \text {, } \\
& \kappa_{111}=\mathbb{E}\left(\frac{\partial^{3} \ell(\theta)}{\partial \gamma^{3}}\right) \text {, } \\
& =\mathbb{E}\left(\frac{2 n}{\gamma^{3}}-4 \sum_{i=1}^{n} \frac{\left(1-x_{i}\right)^{3 \delta}}{\left(1-(1-\gamma)\left(1-x_{i}\right)^{\delta}\right)^{3}}\right) \text {, } \\
& =\frac{2 n}{\gamma^{3}}-4 \sum_{i=1}^{n}\left(\int_{0}^{1} \frac{\left(1-x_{i}\right)^{3 \delta}}{\left(1-(1-\gamma)\left(1-x_{i}\right)^{\delta}\right)^{3}} \cdot \frac{\gamma \delta\left(1-x_{i}\right)^{\delta-1}}{\left(1-(1-\gamma)\left(1-x_{i}\right)^{\delta}\right)^{2}} d x_{i}\right) \text {, } \\
& =\frac{2 n}{\gamma^{3}}-4 \sum_{i=1}^{n}\left(\int_{0}^{1} \frac{\gamma \delta\left(1-x_{i}\right)^{4 \delta-1}}{\left(1-(1-\gamma)\left(1-x_{i}\right)^{\delta}\right)^{5}} d x_{i}\right) \text {, } \\
& =\frac{2 n}{\gamma^{3}}-4 \gamma \delta \sum_{i=1}^{n} \sum_{m=0}^{\infty}\left(\frac{\Gamma(m+5)}{\Gamma(m+1) \Gamma(5)} \cdot(1-\gamma)^{m} \int_{0}^{1}\left(1-x_{i}\right)^{\delta(m+4)-1} d x_{i}\right) \text {, } \\
& =\frac{2 n}{\gamma^{3}}-4 \gamma n \sum_{m=0}^{\infty}\left(\frac{\Gamma(m+5)}{\Gamma(m+1) \Gamma(5)} \cdot \frac{(1-\gamma)^{m}}{(m+4)}\right)(\text { Equation (13)), }
\end{aligned}
$$




$$
\begin{aligned}
& \kappa_{112}=\mathbb{E}\left(\frac{\partial^{3} \ell(\theta)}{\partial \gamma^{2} \partial \delta}\right) \\
& =\mathbb{E}\left(4 \sum_{i=1}^{n} \frac{\left(1-x_{i}\right)^{2 \delta} \log \left(1-x_{i}\right)}{\left(1-(1-\gamma)\left(1-x_{i}\right)^{\delta}\right)^{3}}\right), \\
& =4 \sum_{i=1}^{n}\left(\int_{0}^{1} \frac{\left(1-x_{i}\right)^{2 \delta} \log \left(1-x_{i}\right)}{\left(1-(1-\gamma)\left(1-x_{i}\right)^{\delta}\right)^{3}} \cdot \frac{\gamma \delta\left(1-x_{i}\right)^{\delta-1}}{\left(1-(1-\gamma)\left(1-x_{i}\right)^{\delta}\right)^{2}} d x_{i}\right) \text {, } \\
& =4 \sum_{i=1}^{n}\left(\int_{0}^{1} \frac{\gamma \delta\left(1-x_{i}\right)^{3 \delta-1} \log \left(1-x_{i}\right)}{\left(1-(1-\gamma)\left(1-x_{i}\right)^{\delta}\right)^{5}} d x_{i}\right) \text {, } \\
& =4 \gamma \delta \sum_{i=1}^{n} \sum_{m=0}^{\infty}\left(\frac{\Gamma(m+5)}{\Gamma(m+1) \Gamma(5)} \cdot(1-\gamma)^{m} \int_{0}^{1}\left(1-x_{i}\right)^{\delta(m+3)-1} \log \left(1-x_{i}\right) d x_{i}\right) \text {, } \\
& =-\frac{4 \gamma n}{\delta} \sum_{m=0}^{\infty}\left(\frac{\Gamma(m+5)}{\Gamma(m+1) \Gamma(5)} \cdot \frac{(1-\gamma)^{m}}{(m+3)^{2}}\right) \text { (Equation (14)), } \\
& \kappa_{122}=\mathbb{E}\left(\frac{\partial^{3} \ell(\theta)}{\partial \gamma \partial \delta^{2}}\right) \text {, } \\
& =\mathbb{E}\left(-2 \sum_{i=1}^{n} \frac{\left(1-x_{i}\right)^{\delta} \log ^{2}\left(1-x_{i}\right)}{\left(1-(1-\gamma)\left(1-x_{i}\right)^{\delta}\right)^{2}}+4 \sum_{i=1}^{n} \frac{(\gamma-1)\left(1-x_{i}\right)^{2 \delta} \log ^{2}\left(1-x_{i}\right)}{\left(1-(1-\gamma)\left(1-x_{i}\right)^{\delta}\right)^{3}}\right), \\
& =-2 \sum_{i=1}^{n}\left(\int_{0}^{1} \frac{\left(1-x_{i}\right)^{\delta} \log ^{2}\left(1-x_{i}\right)}{\left(1-(1-\gamma)\left(1-x_{i}\right)^{\delta}\right)^{2}} \cdot \frac{\gamma \delta\left(1-x_{i}\right)^{\delta-1}}{\left(1-(1-\gamma)\left(1-x_{i}\right)^{\delta}\right)^{2}} d x_{i}\right) \\
& +4 \sum_{i=1}^{n}\left(\int_{0}^{1} \frac{(\gamma-1)\left(1-x_{i}\right)^{2 \delta} \log ^{2}\left(1-x_{i}\right)}{\left(1-(1-\gamma)\left(1-x_{i}\right)^{\delta}\right)^{3}} \cdot \frac{\gamma \delta\left(1-x_{i}\right)^{\delta-1}}{\left(1-(1-\gamma)\left(1-x_{i}\right)^{\delta}\right)^{2}} d x_{i}\right), \\
& =-2 \sum_{i=1}^{n}\left(\int_{0}^{1} \frac{\gamma \delta\left(1-x_{i}\right)^{2 \delta-1} \log ^{2}\left(1-x_{i}\right)}{\left(1-(1-\gamma)\left(1-x_{i}\right)^{\delta}\right)^{4}} d x_{i}\right) \text {, } \\
& +4 \sum_{i=1}^{n}\left(\int_{0}^{1} \frac{\gamma \delta(\gamma-1)\left(1-x_{i}\right)^{3 \delta-1} \log ^{2}\left(1-x_{i}\right)}{\left(1-(1-\gamma)\left(1-x_{i}\right)^{\delta}\right)^{5}} d x_{i}\right) \text {, } \\
& =-2 \gamma \delta \sum_{i=1}^{n} \sum_{m=0}^{\infty}\left(\frac{\Gamma(m+4)}{\Gamma(m+1) \Gamma(4)} \cdot(1-\gamma)^{m} \int_{0}^{1}\left(1-x_{i}\right)^{\delta(m+2)-1} \log ^{2}\left(1-x_{i}\right) d x_{i}\right), \\
& -4 \gamma \delta(1-\gamma) \sum_{i=1}^{n} \sum_{m=0}^{\infty}\left(\frac{\Gamma(m+5)}{\Gamma(m+1) \Gamma(5)} \cdot(1-\gamma)^{m} \int_{0}^{1}\left(1-x_{i}\right)^{\delta(m+3)-1} \log ^{2}\left(1-x_{i}\right) d x_{i}\right), \\
& =-\frac{4 \gamma n}{\delta^{2}} \sum_{m=0}^{\infty}\left(\frac{\Gamma(m+4)}{\Gamma(m+1) \Gamma(4)}\left(\frac{(1-\gamma)^{m}}{(m+2)^{3}}+\frac{(m+4)(1-\gamma)^{m+1}}{2(m+3)^{3}}\right)\right)(\text { Equation (15)), }
\end{aligned}
$$




$$
\begin{aligned}
& \kappa_{222}=\mathbb{E}\left(\frac{\partial^{3} \ell(\theta)}{\partial \delta^{3}}\right) \\
& =\mathbb{E}\left(\frac{2 n}{\delta^{3}}+2 \sum_{i=1}^{n} \frac{(1-\gamma)\left(1-x_{i}\right)^{\delta} \log ^{3}\left(1-x_{i}\right)}{\left(1-(1-\gamma)\left(1-x_{i}\right)^{\delta}\right)^{2}}+4 \sum_{i=1}^{n} \frac{(1-\gamma)^{2}\left(1-x_{i}\right)^{2 \delta} \log ^{3}\left(1-x_{i}\right)}{\left(1-(1-\gamma)\left(1-x_{i}\right)^{\delta}\right)^{3}}\right), \\
& =\frac{2 n}{\delta^{3}}+2 \sum_{i=1}^{n}\left(\int_{0}^{1} \frac{(1-\gamma)\left(1-x_{i}\right)^{\delta} \log ^{3}\left(1-x_{i}\right)}{\left(1-(1-\gamma)\left(1-x_{i}\right)^{\delta}\right)^{2}} \cdot \frac{\gamma \delta\left(1-x_{i}\right)^{\delta-1}}{\left(1-(1-\gamma)\left(1-x_{i}\right)^{\delta}\right)^{2}} d x_{i}\right) \\
& +4 \sum_{i=1}^{n}\left(\int_{0}^{1} \frac{(1-\gamma)^{2}\left(1-x_{i}\right)^{2 \delta} \log ^{3}\left(1-x_{i}\right)}{\left(1-(1-\gamma)\left(1-x_{i}\right)^{\delta}\right)^{3}} \cdot \frac{\gamma \delta\left(1-x_{i}\right)^{\delta-1}}{\left(1-(1-\gamma)\left(1-x_{i}\right)^{\delta}\right)^{2}} d x_{i}\right), \\
& =\frac{2 n}{\delta^{3}}+2 \sum_{i=1}^{n}\left(\int_{0}^{1} \frac{\gamma \delta(1-\gamma)\left(1-x_{i}\right)^{2 \delta-1} \log ^{3}\left(1-x_{i}\right)}{\left(1-(1-\gamma)\left(1-x_{i}\right)^{\delta}\right)^{4}} d x_{i}\right) \\
& +4 \sum_{i=1}^{n}\left(\int_{0}^{1} \frac{\gamma \delta(1-\gamma)^{2}\left(1-x_{i}\right)^{3 \delta-1} \log ^{3}\left(1-x_{i}\right)}{\left(1-(1-\gamma)\left(1-x_{i}\right)^{\delta}\right)^{5}} d x_{i}\right) \\
& =\frac{2 n}{\delta^{3}}+2 \gamma \delta(1-\gamma) \sum_{i=1}^{n} \sum_{m=0}^{\infty}\left(\frac{\Gamma(m+4)}{\Gamma(m+1) \Gamma(4)} \cdot(1-\gamma)^{m} \int_{0}^{1}\left(1-x_{i}\right)^{\delta(m+2)-1} \log ^{3}\left(1-x_{i}\right) d x_{i}\right) \\
& +4 \gamma \delta(1-\gamma)^{2} \sum_{i=1}^{n} \sum_{m=0}^{\infty}\left(\frac{\Gamma(m+5)}{\Gamma(m+1) \Gamma(5)} \cdot(1-\gamma)^{m} \int_{0}^{1}\left(1-x_{i}\right)^{\delta(m+3)-1} \log ^{3}\left(1-x_{i}\right) d x_{i}\right), \\
& =\frac{2 n}{\delta^{3}}+\frac{12 \gamma n}{\delta^{3}}\left(\frac{\Gamma(m+4)}{\Gamma(m+1) \Gamma(4)}\left(\frac{(1-\gamma)^{m+1}}{(m+2)^{4}}+\frac{(m+4)(1-\gamma)^{m+2}}{2(m+3)^{4}}\right)\right)(\text { Equation }(16)) \text {. }
\end{aligned}
$$

